\title{
Necessity of electrically conductive pili for methanogenesis with magnetite stimulation
}

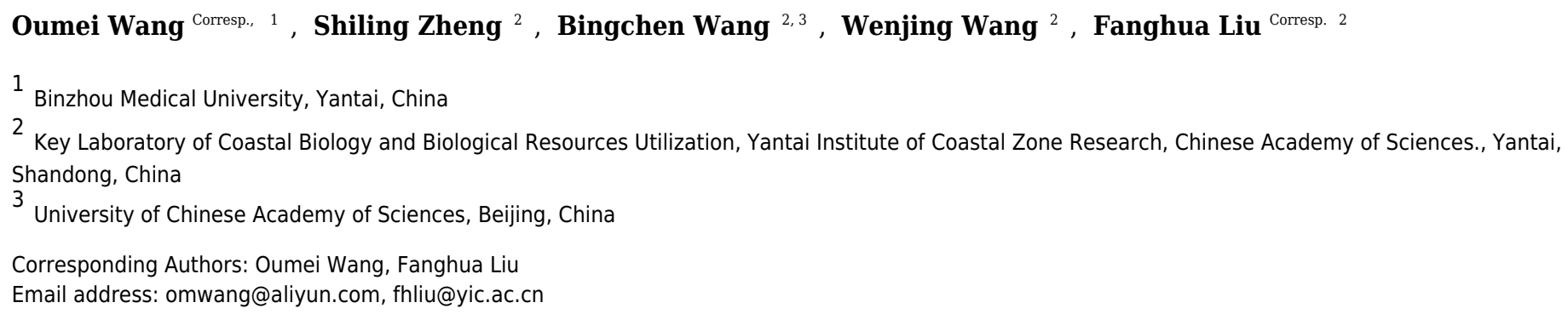

Background: Magnetite-mediated direct interspecies electron transfer (DIET) between Geobacter and Methanosarcina species is increasingly being invoked to explain magnetite stimulation of methane production in anaerobic soils and sediments. Although magnetitemediated DIET has been documented in defined co-cultures reducing fumarate or nitrate as the electron acceptor, the effects of magnetite have only been inferred in methanogenic systems. Methods: Concentrations of methane and organic acid were analysed with gas chromatograph and high-performance liquid chromatography, respectively. The concentration of $\mathrm{HCl}$-extractable $\mathrm{Fe}(\mathrm{II})$ was determined by the ferrozine method. The association of the defined co-cultures of $G$. metallireducens and $M$. barkeri with magnetite was observed with transmission electron micrographs. Results: Magnetite stimulated ethanol metabolism and methane production in defined co-cultures of $G$. metallireducens and $M$. barkeri; however, magnetite did not promote methane production in co-cultures initiated with a culture of $G$. metallireducens that could not produce electrically conductive pili (e-pili), unlike the conductive carbon materials that facilitate DIET in the absence of e-pili. Transmission electron microscopy revealed that $G$. metallireducens and $M$. barkeri were closely associated when magnetite was present, as previously observed in $G$. metallireducens/G. sulfurreducens co-cultures. These results show that magnetite can promote DIET between Geobacter and Methanosarcina species, but not as a substitute for e-pili, and probably functions to facilitate electron transfer from the e-pili to Methanosarcina. Conclusion: In summary, the e-pili are necessary for the stimulation of not only $G$. metallireducens/G. sulfurreducens, but also methanogenic $G$. metallireducens/M. barkeri co-cultures with magnetite. 


\section{Necessity of electrically conductive pili for methanogenesis with}

\section{2 magnetite stimulation}

3

4 Oumei Wang ${ }^{1, *}$, Shiling Zheng ${ }^{2}$, Bingchen Wang ${ }^{2,3}$, Wenjing Wang ${ }^{2}$, Fanghua Liu ${ }^{2, *}$ 5

6 Binzhou Medical University, Yantai, China; ${ }^{1}$ Key Laboratory of Coastal Biology and Biological

7 Resources Utilization, Yantai Institute of Coastal Zone Research, Chinese Academy of Sciences, 8 Yantai, China; ${ }^{3}$ University of Chinese Academy of Sciences, Beijing, China.

$10 *$ Corresponding author

12 Oumei Wang, Binzhou Medical University, 346 Guanhai Road, Laishan District, Yantai,

13 Shandong 264003, China. Email: omwang@aliyun.com.

14 or Fanghua Liu, Yantai Institute of Coastal Zone Research, 17 Chunhui Road, Laishan District,

15 Yantai, Shandong 264003, China. Email: fhliu@yic.ac.cn.

16

17 Running title: E-pili for magnetite stimulation 


\section{ABSTRACT}

19 Background: Magnetite-mediated direct interspecies electron transfer (DIET) between

Geobacter and Methanosarcina species is increasingly being invoked to explain magnetite stimulation of methane production in anaerobic soils and sediments. Although magnetitemediated DIET has been documented in defined co-cultures reducing fumarate or nitrate as the electron acceptor, the effects of magnetite have only been inferred in methanogenic systems.

Methods: Concentrations of methane and organic acid were analysed with gas chromatograph and high-performance liquid chromatography, respectively. The concentration of HCl-extractable $\mathrm{Fe}(\mathrm{II})$ was determined by the ferrozine method.

The association of the defined co-cultures of G. metallireducens and M. barkeri with magnetite was observed with transmission electron micrographs.

Results: Magnetite stimulated ethanol metabolism and methane production in defined cocultures of G. metallireducens and M. barkeri; however, magnetite did not promote methane production in co-cultures initiated with a culture of $G$. metallireducens that could not produce electrically conductive pili (e-pili), unlike the conductive carbon materials that facilitate DIET in the absence of e-pili. Transmission electron microscopy revealed that G. metallireducens and $M$. barkeri were closely associated when magnetite was present, as previously observed in $G$. metallireducens/G. sulfurreducens co-cultures. These results show that magnetite can promote DIET between Geobacter and Methanosarcina species, but not as a substitute for e-pili, and probably functions to facilitate electron transfer from the e-pili to Methanosarcina.

Conclusion: In summary, the e-pili are necessary for the stimulation of not only $G$. metallireducens/G. sulfurreducens, but also methanogenic G. metallireducens/M. barkeri cocultures with magnetite.

\section{INTRODUCTION}


44 Microbial methane production is one of the most successful, large-scale bioenergy strategies (Liu 45 et al., 2009; Shen et al., 2016) and methane production in terrestrial environments is a major 46 source of atmospheric methane, an important greenhouse gas (Bridgham et al., 2013; Conrad 47 2007). In freshwater methanogenic environments, and anaerobic digesters, methanogens 48 primarily produce methane from the metabolism of acetate and the reduction of carbon dioxide 49 with $\mathrm{H}_{2}$ to methane. The well-known source of electrons for carbon dioxide reduction to methane 50 is $\mathrm{H}_{2}$ (Sieber et al., 2012); however, it has recently been demonstrated that Methanosaeta and 51 Methanosarcina species can accept electrons from the donor strain G. metallireducens for carbon 52 dioxide reduction via direct interspecies electron transfer (DIET) (Chen et al., 2014a; Chen et al., $532014 b$; Rotaru et al., 2014a; Rotaru et al., 2014b; Wang et al., 2016).

54 In the absence of added conductive materials, DIET between Geobacter metallireducens and Methanosaeta and Methanosarcina species requires the electrically conductive pili (e-pili) of G. metallireducens (Chen et al., 2014a; Rotaru et al., 2014a; Rotaru et al., 2014b). The e-pili of both Geobacter species are also required for DIET in co-cultures of G. metallireducens and G. sulfurreducens (Shrestha et al., 2009; Summers et al., 2010). Existing studies on the e-pili of G. sulfurreducens have suggested that the conductivity along the length of Geobacter e-pili (Adhikari et al., 2016; Malvankar \& Lovley 2014) can be attributed to the tight packing of aromatic amino acids within the pilus structure, which confer a metallic-like conductivity similar to that observed in carbon nanotubes (Malvankar et al., 2015; Malvankar et al., 2011; Vargas et al., 2013). The e-pili are decorated with the $c$-type cytochrome OmcS, which does not contribute to conductivity along the length of the e-pili, but is important for electron transfer from the e-pili to extracellular electron acceptors/donors (Leang et al., 2010; Liu et al., 2015; Malvankar \& Lovley 2014; Malvankar et al., 2012; Mehta et al., 2005; Summers et al., 2010). It is expected that the e-pili of G. metallireducens function in a similar manner(Smith et al., 2013; Tremblay et al., 2012; Zheng et al., 2017), but the cytochrome(s) that are attached to the e-pili of $G$. metallireducens have not yet been identified. 
Conductive carbon materials, such as: granular activated carbon, carbon cloth, and biochar,

71 stimulate DIET (Chen et al., 2014a; Chen et al., 2014b; Liu et al., 2012; Rotaru et al., 2014a).

72 The electron-donating and electron-accepting partners both attach to the conductive carbon materials, which serve as an electrical conduit between the two species. Mutant Geobacter strains that lack e-pili can participate in DIET under these conditions, presumably because biological cell-to-cell electrical conduits are no longer required (Chen et al., 2014a; Chen et al., 2014b; Liu et al., 2012; Rotaru et al., 2014a).

An important insight into carbon and electron flow in methanogenic environments lies in the finding that magnetite stimulated methane production in enrichment cultures inoculated from paddy soil with either ethanol or acetate as the electron donor (Kato et al., 2012a). The enhanced methane production was accompanied by an enrichment of Geobacter and Methanosarcina species (Kato et al., 2012a). It was hypothesised that the magnetite provided electrical contact between the Geobacter and Methanosarcina species and that the Geobacter species oxidized the ethanol or acetate to carbon dioxide with electron transfer to the Methanosarcina, which then used the electrons to reduce carbon dioxide to methane (Kato et al., 2012a). Many subsequent studies have documented the fact that magnetite accelerates methane production in samples from sediments or anaerobic digesters or defined co-cultures and have also inferred that this can be attributed to enhanced electron transfer through magnetite to methanogens (Li et al., 2015; Tang et al., 2016; Yang et al., 2015; Zhuang et al., 2015). Magnetite does promote interspecies

89 electron transfer between Geobacter sulfurreducens and Thiobacillus denitrificans growing with 90 acetate as the electron donor and nitrate as the electron acceptor (Kato et al., 2012b), as well as

91 between G. metallirducens and G. sulfurreducens growing with ethanol as the electron donor and 92 fumarate as the electron acceptor (Liu et al., 2015)., however, it has never been directly 93 demonstrated that magnetite promotes DIET to methanogens. Analysis of the mechanisms by 94 which magnetite enhanced DIET in G. metallireducens/G. sulfurreducens co-cultures indicated 95 that, unlike conductive carbon materials, magnetite does not act as a substitute for e-pili, but 
96 rather can take the place of OmcS by attaching to e-pili to facilitate DIET, thus alleviating the

97 need for OmcS production (Liu et al., 2015). Therefore, it should not be assumed that magnetite

98 promotes DIET to methanogens as has been demonstrated for conductive carbon materials. The

99 purpose of this study was to evaluate further the possibility that magnetite promotes DIET to

100 methanogens.

\section{MATERIALS AND METHODS}

\section{Microorganisms, media, and growth conditions}

103 Wild-type Geobacter metallireducens strain GS-15 (ATCC 53774) (Aklujkar et al., 2009; Lovley

104 et al., 1993) and a strain of G. metallireducens in which the gene for PilA, the pilus monomer,

105 was deleted (Tremblay et al., 2012) were obtained from our laboratory collection.

106 Methanosarcina barkeri strain DSM 800 (ATCC 43569) was obtained from DSMZ

107 (Braunschweig, Germany).

108 All culturing was performed under strict anaerobic conditions under a gas phase of $\mathrm{N}_{2} / \mathrm{CO}_{2}$

109 (80/20). Inocula for co-cultures were developed by growing G. metallireducens strains in Fe(III)-

110 citrate (FC) medium (Bagnara et al., 1985), with $20 \mathrm{mmol} \mathrm{L}^{-1}$ ethanol as the sole electron donor

111 and $55 \mathrm{mmol} \mathrm{L}^{-1} \mathrm{Fe}(\mathrm{III})$ citrate as the electron acceptor. For co-cultures of G. metallireducens

112 and M. barkeri, G. metallireducens was grown in DSMZ methanogenic medium 120 with 20

$113 \mathrm{mmol} \mathrm{L}-1$ ethanol as the electron donor and nitrate $\left(10 \mathrm{mmol} \mathrm{L}^{-1}\right)$ as the electron acceptor. $M$.

114 barkeri was grown in the same medium with $30 \mathrm{mmol} \mathrm{L}^{-1}$ acetate as the substrate. Co-cultures

115 were grown in $40 \mathrm{~mL}$ medium 120 with a $10 \%$ inoculum and with ethanol $\left(20 \mathrm{mmol} \mathrm{L}^{-1}\right)$ as the

116 electron donor as described previously (Rotaru et al., 2014a). The incubation temperature for all

117 methanogenic studies was $37^{\circ} \mathrm{C}$. When noted, magnetite was prepared as previously described

118 (Kang et al., 1996) and added from stock solutions to give a final concentration of $5 \mathrm{mmol} \mathrm{L}^{-1}$

119 before autoclaving.

\section{Chemical analysis}


121 The gaseous samples were regularly collected from enrichment cultures with pressure-lock

122 analytical syringes. The concentrations of $\mathrm{CH}_{4}$ were analysed with a gas chromatograph (GC-

123 7890A; Agilent Technologies, USA) equipped with a flame ionisation detector.

124 Concentrations of ethanol and acetate were analysed with high-performance liquid

125 chromatography (1260 Infinity; Agilent Technologies, USA) with a Hi-plex H column equipped

126 with a refractive index detector.

127 The concentration of HCl-extractable $\mathrm{Fe}(\mathrm{II})$ was extracted from cultures and each replicate

128 of the assays in triplicate as described previously (Zheng et al., 2015). Moreover, the

129 concentration of dissolved Fe(II) in samples was also quantified by filtering through $0.45 \mu \mathrm{m}$

130 sterile syringe filters and using the ferrozine method as described above.

131 Microscopy

132 Samples of cells and magnetite were negatively stained with $2 \%$ phosphotungstic acid and

133 examined by a JEM-1400 (JEOL, Japan) transmission electron microscope (TEM).

\section{RESULTS AND DISCUSSION}

\section{Magnetite stimulation of DIET between $\boldsymbol{G}$. metallireducens and M. barkeri}

136 To evaluate whether, or not, magnetite was capable of stimulating DIET between $G$.

137 metallireducens and M. barkeri, co-cultures were initiated with ethanol as the sole electron donor

138 in the presence, and absence, of magnetite. Although M. bakeri is capable of using $\mathrm{H}_{2}$ as an

139 electron donor, G. metallireducens cannot metabolise ethanol with the production of $\mathrm{H}_{2}$ (Rotaru

140 et al., 2014b; Shrestha et al., 2013a; Summers et al., 2010) and thus syntrophic growth in $G$.

141 metallireducens/M. barkeri co-cultures can be attributed to DIET. First, based on Fig. 1A, the

142 production of $\mathrm{CH}_{4}$ from the co-culture of $G$. metallireducens and M. barkeri without magnetite

143 indeed indicates DIET in syntrophic interaction. However, DIET is not exclusively occurred, at

144 least a part of methane was still produced from acetate. For example, if half of the methane

$145(\sim 0.25 \mathrm{mmol})$ was produced from acetate $(\sim 0.25 \mathrm{mmol})$, the concentration of acetate remained in 
146 the medium should be $\sim 0.25 \mathrm{mmol}$.

147 The initial establishment of G. metallireducens and M. barkeri co-cultures requires a long

148 adaption period in the absence of added conductive materials (Rotaru et al., 2014a). As expected,

149 ethanol was only slowly metabolised over 50 days without magnetite (Fig. 1C), however, in the

150 presence of magnetite, ethanol was metabolised with the production of methane beginning within

15110 days (Fig. 1C). Non-inoculated controls with magnetite showed no ethanol metabolism or

152 methane production.

153 Limited acetate accumulated in the G. metallireducens with, or without, M. barkeri co-

154 cultures in the presence of magnetite $\left(\mathrm{C}_{2} \mathrm{H}_{6} \mathrm{O}+\mathrm{H}_{2} \mathrm{O} \rightarrow \mathrm{C}_{2} \mathrm{H}_{4} \mathrm{O}_{2}+4 \mathrm{H}^{+}+4 \mathrm{e}^{-}\right.$, Oxidation of one

155 ethanol will produce one acetate plus four electrons released (Rotaru et al., 2014a)), but was

156 later consumed (Fig. 1D), which differed from co-cultures of G. metallireducens and M. barkeri

157 in the absence of magnetite, suggesting that $G$. metallireducens metabolised the acetate that $G$.

158 metallireducens produced from ethanol compared with the result of $G$. metallireducens acting

159 alone with magnetite. The high amount of methane in the G. metallireducens and M. barkeri co-

160 cultures suggested that M. barkeri only used the electrons released from ethanol oxidation for

161 reducing carbon dioxide to produce methane in the magnetite-amended co-cultures $\left(8 \mathrm{H}^{+}+8 \mathrm{e}^{-}+\right.$

$\left.162 \mathrm{CO}_{2} \rightarrow \mathrm{CH}_{4}+2 \mathrm{H}_{2} \mathrm{O}\right)$. The total amount of ethanol from the magnetite-amended co-cultures

163 metabolised $(1.15 \pm 0.12 \mathrm{mmol})$ resulted in $1.60 \pm 0.0032 \mathrm{mmol}$ methane (Figs $1 \mathrm{~A}, \mathrm{C})$, which

164 showed that the mmol ratio of $\mathrm{CH}_{4} / \mathrm{C}_{2} \mathrm{H}_{6} \mathrm{O}(1.60 / 1.15)$ was $1.39(>1)$, thus about $92.2 \%$ of the

165 electrons from ethanol oxidation were recovered in methane according to the equation: $2 \mathrm{C}_{2} \mathrm{H}_{5} \mathrm{OH}$

$166 \rightarrow 3 \mathrm{CH}_{4}+\mathrm{CO}_{2}$. Furthermore, no $\mathrm{H}_{2}$ was detected in any of the experiment groups. This result

167 was consistent with the fact that $G$. metallireducens is unable to produce $\mathrm{H}_{2}$ during ethanol

168 metabolism (Shrestha et al., 2013b). Therefore, the high electron recovery that was available

169 from ethanol to methane suggested that magnetite can stimulate DIET between $G$.

170 metallireducens and M. barkeri and suggested that the simplest explanation for the enrichment of

171 Geobacter and Methanosarcina observed in the presence of magnetite in previous studies (Kato 
172 et al., 2012a) is that magnetite was facilitating DIET.

173 HCl-extractable ferrous iron was also produced in G. metallireducens-M. barkeri co-

174 cultures from reduction ferric iron of magnetite within five days and increased to $0.1768 \pm$

$1750.0219 \mathrm{mmol}$ at 50 days, which was equal to that when G. metallireducens was tested with

176 magnetite alone $(0.1761 \pm 0.0549 \mathrm{mmol})$ (Fig. 1B); however, the concentration of dissolved

177 ferrous iron was under detect limitation during the incubation of the co-cultures amended with

178 magnetite, suggested that only a part of the ferric iron in the added magnetite was reduced to

179 ferrous iron. The results indicated that only a small portion of electrons (about $4.6 \mathrm{mmol}$ electrons

180 released from $1.15 \mathrm{mmol}$ ethanol oxidation, $0.18 \mathrm{mmol} / 4.6 \mathrm{mmol}$, about $3.9 \%$ ) in $G$.

181 metallireducens-M. barkeri co-cultures with magnetite were used for ferric iron reduction and

182 the majority of electrons (about 96.1\%) were used for methane production. This result differs

183 from that reporting that magnetite acts as the electrical conduit between electron-donating

184 Geobacter and electron-accepting methanogens (Kato et al., 2012a; Li et al., 2015; Viggi et al.,

185 2014). One factor controlling ferrous iron production in co-cultures amended with magnetite is

186 the range of substrates that can be metabolised by Geobacter species. G. metallireducens can

187 utilise ethanol and acetate, ferrous iron production from acetate was slower than that from

188 ethanol within 10 days in the presence of magnetite, while ferrous iron production from $G$.

189 sulfurreducens amended with magnetite was much lower than that from G. metallireducens

190 when utilising acetate (Fig. 2). This suggested that G. metallireducens, like some

191 microorganisms (e.g., Shewanella, Dechloromonas, Desulfovibrio, and Clostridium) was able to

192 use magnetite as the electron acceptor from ethanol or acetate metabolism (Kostka \& Nealson

193 1995; Yang et al., 2015). However, it is not possible for magnetite to act as the electron shuttle

194 for production of methane from carbon dioxide because of the relatively high mid-point potential 195 of the $\mathrm{Fe}(\mathrm{III}) / \mathrm{Fe}(\mathrm{II})$ redox couple $\left(E_{0}{ }^{\prime}=+0.20 \mathrm{~V}, \mathrm{pH} 7.0\right)$ which is too high for the reduction of 196 carbon dioxide to methane $\left(E_{0}^{\prime}\right.$ of $\mathrm{CO}_{2} /$ methane couple $\left.=-240 \mathrm{mV}\right)$.

197 To determine the actual role of magnetite in stimulation of ethanol metabolism and methane 
198 production in co-cultures of wild-type G. metallireducens and M. barkeri, co-cultures were 199 initiated with $2.5 \mathrm{mmol} \mathrm{L}^{-1}$ magnetite, after a 10-day incubation period, an additional $2.5 \mathrm{mmol}$ $200 \mathrm{~L}^{-1}$ magnetite was subsequently added. Methane production presented the same tendency with 5 $201 \mathrm{mmol} \mathrm{L}^{-1}$ magnetite added to the co-cultures (Fig. 1A): this meant that the manner and amount of 202 addition of magnetite could not affect methane production, however, the amount of $\mathrm{HCl}$ 203 extractable ferrous iron changed: the reduced ferrous iron concentration was about 0.0193-0.0239 204 mmol $\left(\sim 9.6-12 \%\right.$ of added $\left.\mathrm{Fe}^{3+}\right)$ when $2.5 \mathrm{mmol} \mathrm{L}^{-1}$ magnetite $\left(\mathrm{Fe}^{3+}: 0.2 \mathrm{mmol}\right)$ was added 205 during the first 10 days, and subsequently reduced when more magnetite was added, the amount 206 of ferrous iron used in each step (total: $0.1635 \pm 0.0313 \mathrm{mmol}$ ) was similar to the addition of 5

207 mmol L-1 magnetite (Fig. 1B). This result was consistent with the observation that $G$.

208 metallireducens alone reduced magnetite to produce ferrous iron (Fig. 1B). Thus, the initial 209 concentration of magnetite determined how much Fe(III) inside was reduced. When high 210 concentration of magnetite $\left(5 \mathrm{mmol} \mathrm{L}^{-1}\right)$ was available, Fe(III) reduction was detected; however,

211 no significant $\mathrm{Fe}(\mathrm{III})$ reduction was found when lower concentration of magnetite $\left(2.5 \mathrm{mmol} \mathrm{L}^{-1}\right)$ 212 was present. $\mathrm{Fe}(\mathrm{III})$ in the magnetite was reduced only when additional magnetite $\left(2.5 \mathrm{mmol} \mathrm{L}^{-1}\right)$ 213 was added. This demonstrated that lower concentration of magnetite could not be preferentially 214 used as the electron acceptor in the co-culture of G. metallireducens and M. barkeri. Similarly, 215 ethanol was stimulated to oxidise and little acetate was transiently accumulated in magnetite 216 upon its step-by-step addition to co-cultures of wild-type G. metallireducens and M. barkeri

217 (Figs 1C, D). The calculation of electron recovery (93.81\%) of electrons available from ethanol

218 in methane in these samples further suggested that M. barkeri was accepting electrons from 219 carbon dioxide reduction via DIET.

220 Transmission electron microscopy (TEM) revealed that G. metallireducens (rod-shaped 221 cells) and M. barkeri (larger size cocci) were associated with each other (Fig. 3A). With higher 222 magnification it was apparent that magnetite was associated with the G. metallireducens pili (Fig. 223 3B), as was previously observed that some of the magnetite was localised along pili and 
224 compensated for the lack of OmcS of G. sulfurreducens in promoting electrical contacts with pili

225 in G. metallireducens/G. sulfurreducens co-cultures (Liu et al. 2015).

\section{Failure of magnetite to compensate for loss of e-pili in G. metallireducens}

227 To investigate further the mechanisms for magnetite stimulation of DIET between $G$.

228 metallireducens and M. barkeri, co-cultures were initiated with the previously described strain of

229 G. metallireducens (Tremblay et al., 2012) that is incapable of producing pili because the gene

230 for PilA, the pilus monomer, has been deleted. As expected from previous studies (Rotaru et al.,

$2312014 a$ ), methane was not produced in co-cultures with the pili-deficient strain of $G$.

232 metallireducens (Fig. 4A), however, co-cultures amended with magnetite produced less methane

233 (about $0.38 \pm 0.025 \mathrm{mmol}$, Fig. 4A). During co-culture testing, ferrous iron concentrations were

234 below $0.1 \mathrm{mmol}$ in magnetite amended cultures (Fig. 4B). Furthermore, co-cultures with the

235 pilA-deficient G. metallireducens failed to metabolise ethanol or produce acetate with, or

236 without, magnetite amendment (Figs 4C, D). These results suggested that magnetite perhaps can

237 partly substitute for pili to participate in DIET of co-cultures resulting from its electrical

238 conductivity; however, magnetite appears to promote DIET by a mechanism that is different than

239 that in conductive carbon materials such as GAC and carbon cloth (Chen et al., 2014a; Liu et al.,

240 2012). In the presence of GAC or carbon cloth the pili-deficient strain of G. metallireducens can

241 transfer electrons to $M$. barkeri because both species attach to the conductive materials, which

242 are much bigger than individual cells. Magnetite particles are typically smaller (at 20-50 nm)

243 than cells and thus are unlikely to provide effective cell-to-cell contacts (Liu et al., 2015). This

244 was evident in previous studies with G. metallireducens/G. sulfurreducens co-cultures in which

245 magnetite was not able to compensate for the lack of e-pili in G. metallireducens (Liu et al.,

246 2015). Multiple lines of evidence, including studies with an OmcS-deficient mutant, suggested

247 that magnetite could serve as the functional equivalent of OmcS, and the $c$-type cytochrome

248 associated with the e-pili of G. sulfurreducens (Liu et al., 2015). Similar genetic experiments are

249 not yet possible with $G$. metallireducens because the cytochrome(s) associated with the $G$. 
250 meatllireducens e-pili have not been identified. However, the finding that magnetite amendments

251 did not permit the growth of the pili-deficient strain of G. metallireducens in co-culture with $M$.

252 barkeri, suggested the magnetite cannot function as an e-pili substitute in all regards. Magnetite

253 was associated with the e-pili in the G. metallireducens/M. barkeri co-cultures. Therefore, it is

254 likely that magnetite also facilitated electron transfer from the G. metallireducens e-pili to $M$.

255 barkeri in the co-cultures.

\section{CONCLUSIONS}

257 In sum, we have established co-cultures of M. barkeri and wild-type G. metallireducens or a

258 strain deficient in the PilA gene with or without magnetite. The results revealed magnetite

259 stimulated ethanol metabolism and methane production in defined co-cultures of $G$.

260 metallireducens and M. barkeri. However, magnetite did not promote methane production in co-

261 cultures of the pilA-deficient G. metallireducens. These results showed that magnetite could not

262 substitute for e-pili to promote DIET between Geobacter and Methanosarcina species, in which

263 the e-pili are necessary for the stimulation.

\section{REFERENCES}

265 Adhikari RY, Malvankar NS, Tuominen MT, and Lovley DR. 2016. Conductivity of individual

266 Geobacter pili. Rsc Advances 6:8363-8366. 10.1039/c5ra28092c

267 Aklujkar M, Krushkal J, DiBartolo G, Lapidus A, Land ML, and Lovley DR. 2009. The genome sequence of Geobacter metallireducens: features of metabolism, physiology and regulation common and dissimilar to Geobacter sulfurreducens. BMC Microbiology 9. 10.1186/14712180-9-109

271

Bagnara C, Toci R, Gaudin C, and Belaich JP. 1985. Isolation and characterization of a cellulolytic microorganism, cellulomonas fermentans sp. nov. International Journal of Systematic Bacteriology 35:502-507.

Bridgham SD, Cadillo-Quiroz H, Keller JK, and Zhuang Q. 2013. Methane emissions from 
275

276

277

278

279

280

281

282

283

284

285

286

287

288

289

290

291

292

293

294

295

296

297

298

299

300

wetlands: biogeochemical, microbial, and modeling perspectives from local to global scales. Global Change Biology 19:1325-1346. 10.1111/gcb.12131

Chen S, Rotaru A-E, Liu F, Philips J, Woodard TL, Nevin KP, and Lovley DR. 2014a. Carbon cloth stimulates direct interspecies electron transfer in syntrophic co-cultures. Bioresource technology 173:82-86. 10.1016/j.biortech.2014.09.009

Chen S, Rotaru A-E, Shrestha PM, Malvankar NS, Liu F, Fan W, Nevin KP, and Lovley DR. 2014b. Promoting interspecies electron transfer with biochar. Scientific Reports 4. $10.1038 /$ srep05019

Conrad R. 2007. Microbial ecology of methanogens and methanotrophs. In: Sparks DL, ed. Advances in Agronomy, Vol 96, 1-63.

Kang YS, Risbud S, Rabolt JF, and Stroeve P. 1996. Synthesis and characterization of nanometer-size $\mathrm{Fe}_{3} \mathrm{O}_{4}$ and $\gamma-\mathrm{Fe}_{2} \mathrm{O}_{3}$ particles. Chemistry of Materials 8:2209-2211. $10.1021 / \mathrm{cm} 960157 \mathrm{j}$

Kato S, Hashimoto K, and Watanabe K. 2012a. Methanogenesis facilitated by electric syntrophy via (semi) conductive iron-oxide minerals. Environmental Microbiology 14:1646-1654. $10.1111 / j .1462-2920.2011 .02611 . x$

Kato S, Hashimoto K, and Watanabe K. 2012b. Microbial interspecies electron transfer via electric currents through conductive minerals. Proceedings of the National Academy of Sciences of the United States of America 109:10042-10046. 10.1073/pnas.1117592109

Kostka JE, and Nealson KH. 1995. Dissolution and reduction of magnetite by bacteria. Environmental Science \& Technology 29:2535-2540. 10.1021/es00010a012

Leang C, Qian X, Mester T, and Lovley DR. 2010. Alignment of the c-type cytochrome OmcS along pili of Geobacter sulfurreducens. Applied and Environmental Microbiology 76:40804084. 10.1128/aem.00023-10

Li H, Chang J, Liu P, Fu L, Ding D, and Lu Y. 2015. Direct interspecies electron transfer accelerates syntrophic oxidation of butyrate in paddy soil enrichments. Environmental 
302 Liu F, Rotaru A-E, Shrestha PM, Malvankar NS, Nevin KP, and Lovley DR. 2012. Promoting

303 direct interspecies electron transfer with activated carbon. Energy \& Environmental Science

304 5:8982-8989. 10.1039/c2ee22459c

305

306

307

308

309

310

311

312

313

314

315

316

317

318

319

320

321

322

323

324

325

326

Liu F, Rotaru A-E, Shrestha PM, Malvankar NS, Nevin KP, and Lovley DR. 2015. Magnetite compensates for the lack of a pilin-associated $c$-type cytochrome in extracellular electron exchange. Environmental Microbiology 17:648-655. 10.1111/1462-2920.12485

Liu FH, Wang SB, Zhang JS, Zhang J, Yan X, Zhou HK, Zhao GP, Zhou ZH. 2009. The structure of the bacterial and archaeal community in a biogas digester as revealed by denaturing gradient gel electrophoresis and 16S rDNA sequencing analysis. Journal of Applied Microbiology 106:952-966. 10.1111/j.1365-2672.2008.04064.x

Lovley DR, Giovannoni SJ, White DC, Champine JE, Phillips EJP, Gorby YA, and Goodwin S. 1993. Geobacter metallireducens gen. nov. sp. nov, a microorganism capable of coupling the complete oxidation of organic-compounds to the reduction of iron and other metals. Archives of Microbiology 159:336-344. 10.1007/bf00290916

Malvankar NS, and Lovley DR. 2014. Microbial nanowires for bioenergy applications. Current Opinion in Biotechnology 27:88-95. 10.1016/j.copbio.2013.12.003

Malvankar NS, Tuominen MT, and Lovley DR. 2012. Lack of cytochrome involvement in longrange electron transport through conductive biofilms and nanowires of Geobacter sulfurreducens. Energy \& Environmental Science 5:8651-8659. 10.1039/c2ee22330a

Malvankar NS, Vargas M, Nevin K, Tremblay P-L, Evans-Lutterodt K, Nykypanchuk D, Martz E, Tuominen MT, and Lovley DR. 2015. Structural basis for metallic-like conductivity in microbial nanowires. Mbio 6. 10.1128/mBio.00084-15

Malvankar NS, Vargas M, Nevin KP, Franks AE, Leang C, Kim B-C, Inoue K, Mester T, Covalla SF, Johnson JP, Rotello VM, Tuominen MT, and Lovley DR. 2011. Tunable metallic-like conductivity in microbial nanowire networks. Nature Nanotechnology 6:573- 
Mehta T, Coppi MV, Childers SE, and Lovley DR. 2005. Outer membrane $c$-type cytochromes required for $\mathrm{Fe}(\mathrm{III})$ and $\mathrm{Mn}(\mathrm{IV})$ oxide reduction in Geobacter sulfurreducens. Applied and Environmental Microbiology 71:8634-8641. 10.1128/aem.71.12.8634-8641.2005

331

332

333

334

335

336

337

338

339

340

341

342

343

344

345

346

347

348

349

350

351

352

Rotaru A-E, Shrestha PM, Liu F, Markovaite B, Chen S, Nevin K, and Lovley D. 2014a. Direct interspecies electron transfer between Geobacter metallireducens and Methanosarcina barkeri. Applied and Environmental Microbiology:4599-4605. 10.1128/aem.00895-14

Rotaru A-E, Shrestha PM, Liu F, Shrestha M, Shrestha D, Embree M, Zengler K, Wardman C, Nevin KP, and Lovley DR. 2014b. A new model for electron flow during anaerobic digestion: direct interspecies electron transfer to Methanosaeta for the reduction of carbon dioxide to methane. Energy \& Environmental Science 7:408-415. 10.1039/c3ee42189a

Shen L, ZhaoQC, Wu X, Li XZ, Li QB, Wang YP. 2016. Interspecies electron transfer in syntrophic methanogenic consortia: From cultures to bioreactors. Renewable \& Sustainable Energy Reviews 54:1358-1367. 10.1016/j.rser.2015.10.102

Shrestha PM, Kube M, Reinhardt R, and Liesack W. 2009. Transcriptional activity of paddy soil bacterial communities. Environmental Microbiology 11:960-970. 10.1111/j.14622920.2008.01821.x

Shrestha PM, Rotaru A-E, Summers ZM, Shrestha M, Liu F, and Lovley DR. 2013a. Transcriptomic and genetic analysis of direct interspecies electron transfer. Applied and Environmental Microbiology 79:2397-2404. 10.1128/aem.03837-12

Shrestha PM, Rotaru AE, Aklujkar M, Liu FH, Shrestha M, Summers ZM, Malvankar N, Flores DC, and Lovley DR. 2013b. Syntrophic growth with direct interspecies electron transfer as the primary mechanism for energy exchange. Environmental Microbiology Reports 5:904910. $10.1111 / 1758-2229.12093$

Sieber JR, McInerney MJ, and Gunsalus RP. 2012. Genomic insights into syntrophy: The paradigm for anaerobic metabolic cooperation. In: Gottesman S, Harwood CS, and 
Schneewind O, eds. Annual Review of Microbiology, Vol 66, 429-452.

354 Smith JA, Lovley DR, and Tremblay P-L. 2013. Outer cell surface components essential for 355 Fe(III) oxide reduction by Geobacter metallireducens. Applied and Environmental Microbiology 79:901-907. 10.1128/aem.02954-12

357

358

359

360

361

362

363

364

365

366

367

368

369

370

371

372

373

374

375

376

377

378

Summers ZM, Fogarty HE, Leang C, Franks AE, Malvankar NS, and Lovley DR. 2010. Direct exchange of electrons within aggregates of an evolved syntrophic coculture of anaerobic bacteria. Science 330:1413-1415. 10.1126/science.1196526

Tang J, Zhuang L, Ma J, Tang Z, Yu Z, and Zhou S. 2016. Secondary mineralization of ferrihydrite affects microbial methanogenesis in Geobacter-Methanosarcina cocultures. Applied and Environmental Microbiology 82:5869-5877.

Tremblay P-L, Aklujkar M, Leang C, Nevin KP, and Lovley D. 2012. A genetic system for Geobacter metallireducens: role of the flagellin and pilin in the reduction of Fe(III) oxide. Environmental Microbiology Reports 4:82-88. 10.1111/j.1758-2229.2011.00305.x

Vargas M, Malvankar NS, Tremblay P-L, Leang C, Smith JA, Patel P, Synoeyenbos-West O, Nevin KP, and Lovley DR. 2013. Aromatic amino acids required for pili conductivity and long-range extracellular electron transport in Geobacter sulfurreducens. Mbio 4. 10.1128/mBio.00105-13

Viggi CC, Rossetti S, Fazi S, Paiano P, Majone M, and Aulenta F. 2014. Magnetite particles triggering a faster and more robust syntrophic pathway of methanogenic propionate degradation. Environmental Science \& Technology 48:7536-7543. 10.1021/es5016789

Wang L-Y, Nevin KP, Woodard TL, Mu B-Z, and Lovley DR. 2016. Expanding the diet for DIET: Electron donors supporting direct interspecies electron transfer (DIET) in defined cocultures. Frontiers in Microbiology 7. 10.3389/fmicb.2016.00236

Yang ZM, Shi XS, Wang CS, Wang L, and Guo RB. 2015. Magnetite nanoparticles facilitate methane production from ethanol via acting as electron acceptors. Scientific Reports 5. $10.1038 /$ srep 16118 
379 Zheng S, Liu F, Li M, Xiao L, and Wang O. 2017. Comparative transcriptomic insights into the 380 mechanisms of electron transfer in Geobacter co-cultures with activated carbon and 381 magnetite. Science China Life Sciences. 10.1007/s11427-017-9177-1

382 Zheng S, Zhang H, Li Y, Zhang H, Wang O, Zhang J, and Liu F. 2015. Co-occurrence of 383 Methanosarcina mazei and Geobacteraceae in an iron (III)-reducing enrichment culture. $384 \quad$ Frontiers in Microbiology 6. 10.3389/fmicb.2015.00941

385 Zhuang L, Xu JL, Tang J, and Zhou SG. 2015. Effect of ferrihydrite biomineralization on 386 methanogenesis in an anaerobic incubation from paddy soil. Journal of Geophysical

387 Research-Biogeosciences 120:876-886. 10.1002/2014jg002893

388 


\section{Figure 1 (on next page)}

Co-cultures of $G$. metallireducens ( $G . m)$ and $M$. barkeri $(M . b)$ with ethanol as the substrate in the presence, or absence, of magnetite $\left(\mathrm{Fe}_{3} \mathrm{O}_{4}\right)$.

Quantities of methane (A), ferrous iron (B), ethanol(C), and acetate (D) in cultures. Data are the means and standard deviation for triplicate cultures. In some instances the standard deviation was less than the size of the symbol. 


\section{Figure 2 (on next page)}

Quantities of ferrous iron in cultures of Geobacter metallireducens (G.m) and Geobacter sulfurreducens (G.s) in the presence of magnetite with ethanol and acetate as the substrates. 


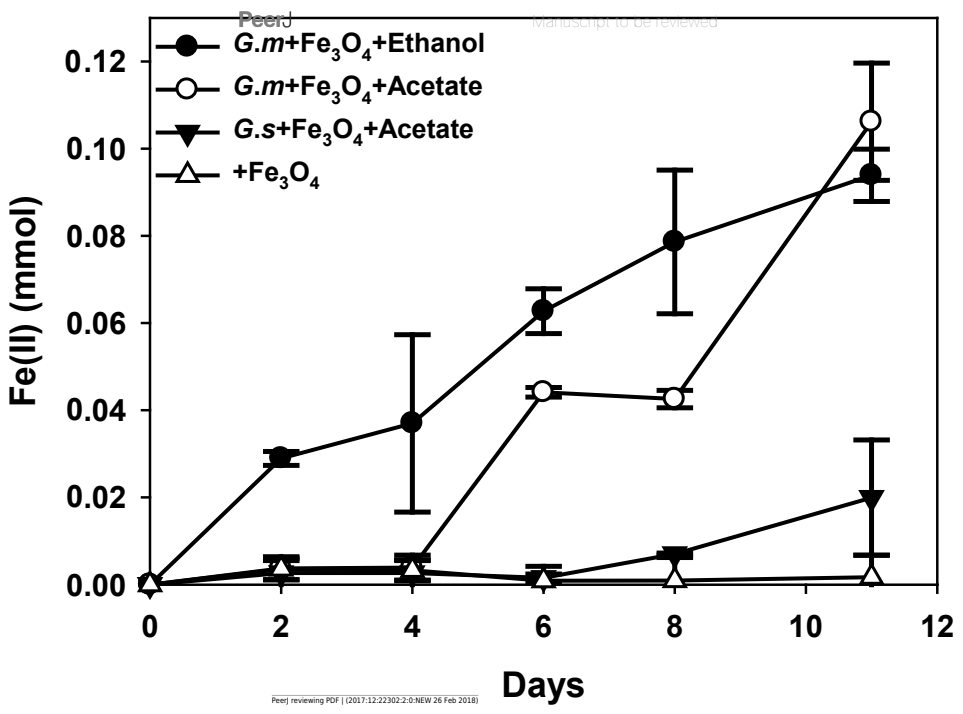


Figure 3 (on next page)

Transmission electron micrographs.

Association of the defined co-cultures of $\mathrm{G}$. metallireducens and M. barkeri with magnetite. (A) Association of the two cell types. Black and white arrows indicate $G$. metallireducens cells and M. barkeri cells, respectively. (B) Association of magnetite with pili. White arrows indicate magnetite and pili. 
PeerJ

$94 \quad-7 d$

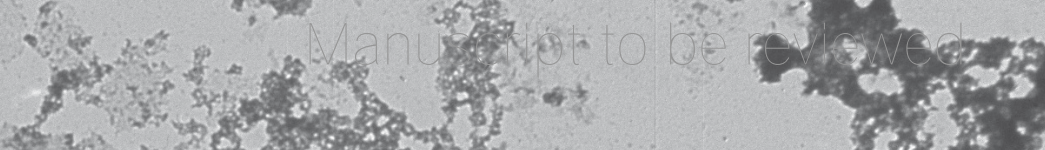
$3+5 ; \div=$

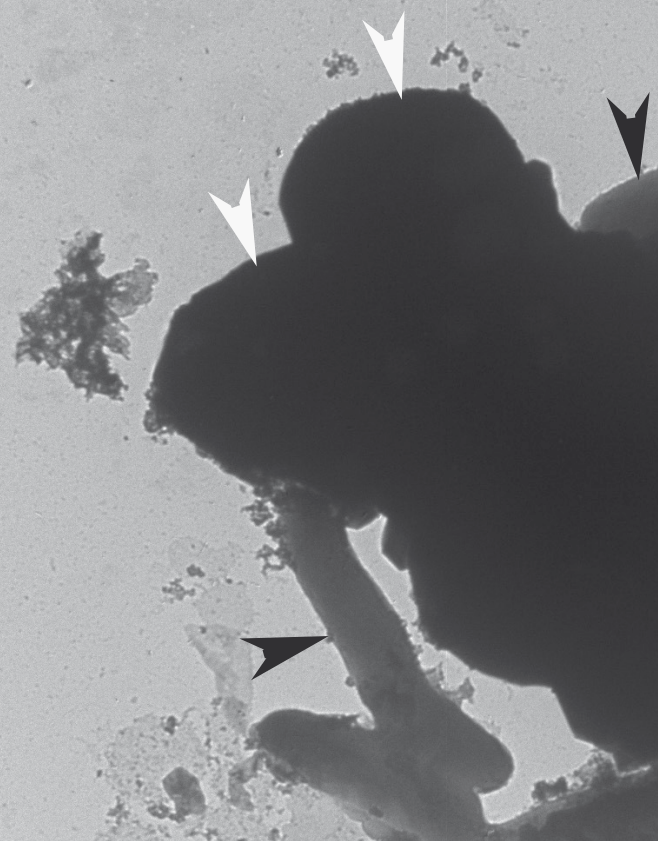

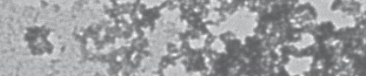

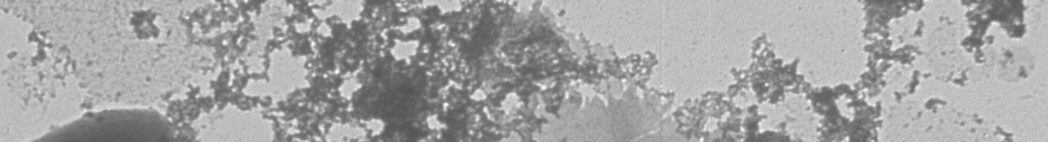
है, 1 है

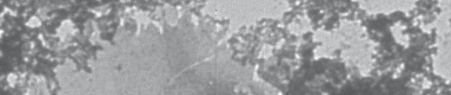

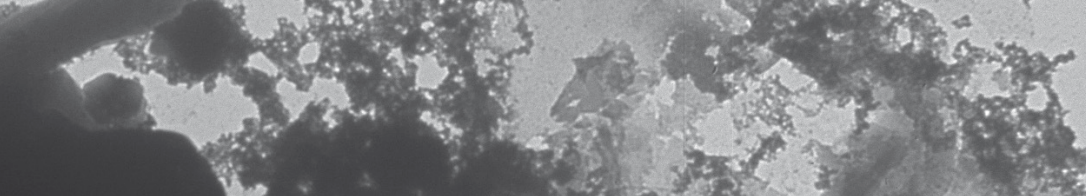

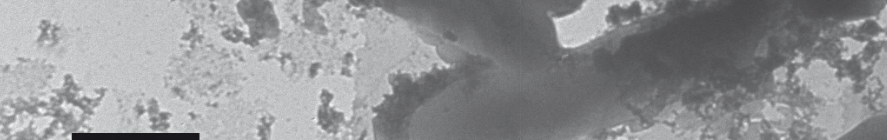

$1000 \mathrm{~nm}$

is.

ลश्र

\&

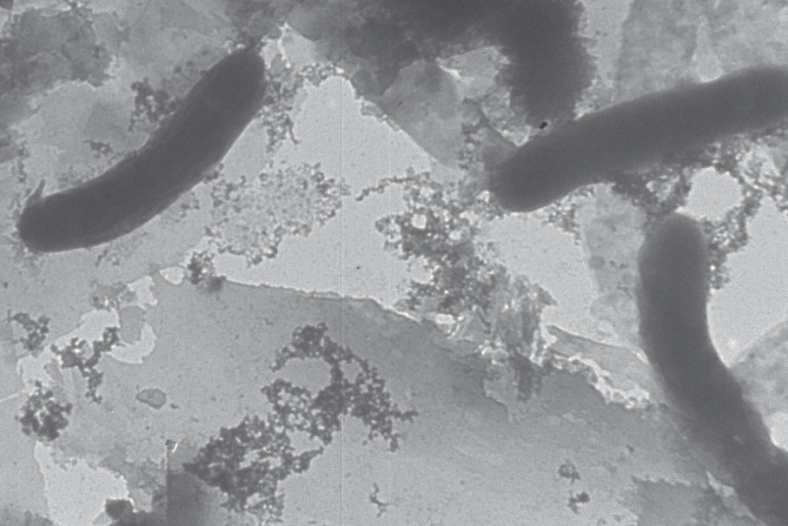

B
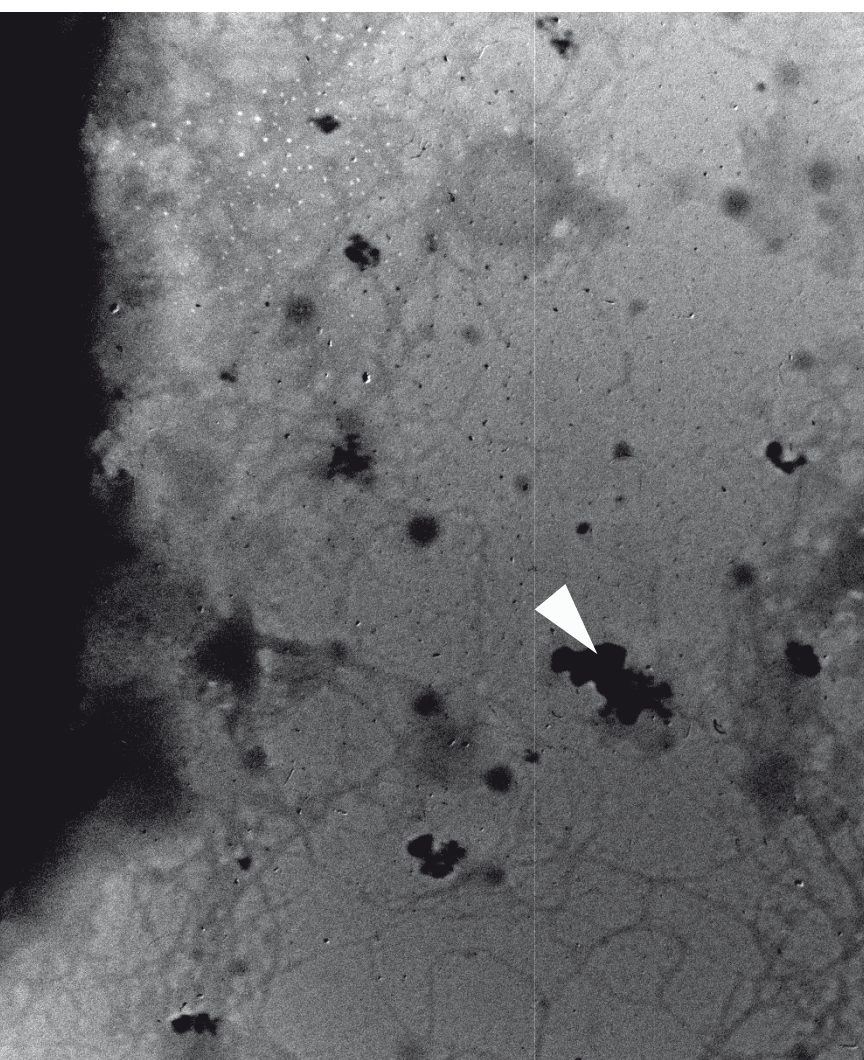

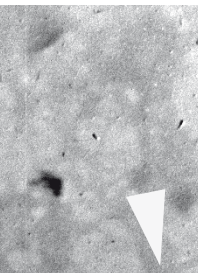

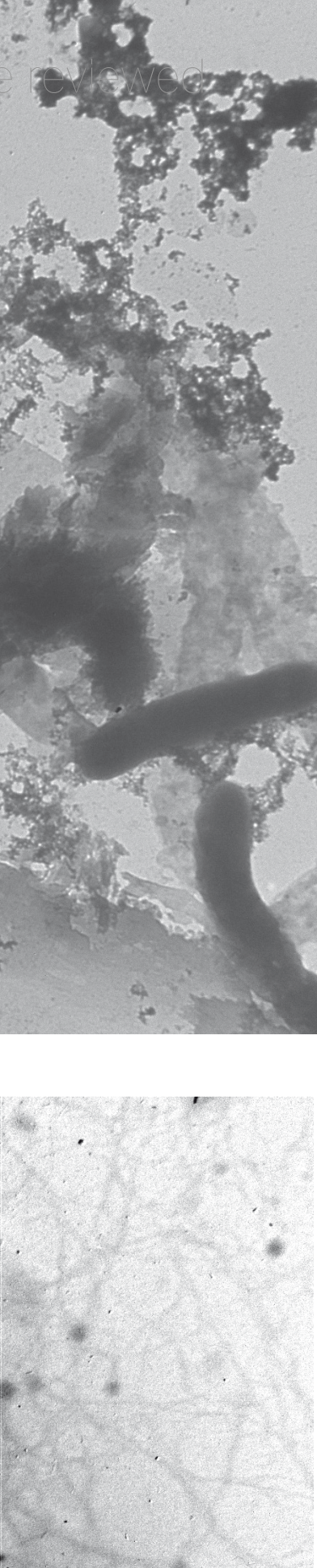

at

.

,

$14 \sin ^{2}$
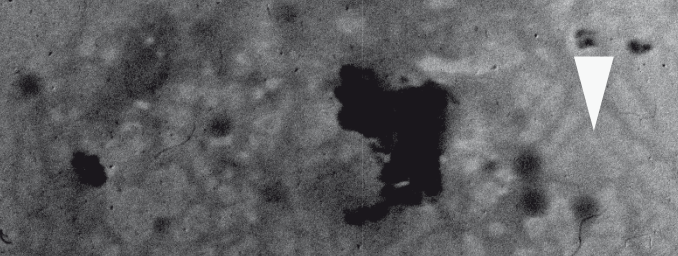

$+$

?

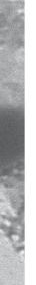


Figure 4(on next page)

Co-cultures of M. barkeri (M. b) and a PilA-deficient G.metallireducens (G. m-deltapilA) strain in the presence, or absence, of magnetite $\left(\mathrm{Fe}_{3} \mathrm{O}_{4}\right)$.

Quantities of methane (A), ferrous iron (B), ethanol(C), and acetate (D) in cultures. Data are the means and standard deviation for triplicate cultures. In some instances the standard deviation was less than the size of the symbol. 
